Proyecciones Journal of Mathematics

Vol. 33, No 1, pp. 43-59, March 2014.

Universidad Católica del Norte

Antofagasta - Chile

\title{
Hardy-Type Spaces and its Dual
}

\author{
René Erlín Castillo \\ Universidad Nacional de Colombia, Colombia \\ and \\ Julio C. Ramos Fernández and Eduard Trousselot \\ Universidad de Oriente, Venezuela \\ Received: August 2012. Accepted : October 2013
}

\begin{abstract}
In this paper we defined a new Hardy-type spaces using atoms on homogeneous spaces which we call $H^{\varphi, q}$. Also we prove that under certain conditions $\mathrm{BMO}_{\varphi}^{(p)}$ is the dual of $H^{\varphi, q}$.
\end{abstract}

Keywords : BMO, Dual space, Hardy space, Space of homogeneous type.

Subjclass [2010] : 32A37; $43 A 85$. 


\section{Introduction}

The Hardy space $H^{p}$ were first studied on the unit disk in the complex plane. In their 1968 paper Duren, Romberg and Shield (see [4]) make the following definitions and comments about $H^{p}$. For $0<p \leq \infty, H^{p}$ is the linear space of functions $f(z)$ analytic in $|z|<1$ such that

$$
M_{p}(r, f)=\left(\frac{1}{2 \pi} \int_{0}^{2 \pi}\left|f\left(r e^{i \theta}\right)\right|^{p} d \theta\right)^{\frac{1}{p}}, \quad 0<p<\infty
$$

or

$$
M_{\infty}(r, f)=\max _{0 \leq \theta<2 \pi}\left|f\left(r e^{i \theta}\right)\right|
$$

remains bounded as $r \rightarrow 1$. If $1 \leq p \leq \infty, H^{p}$ is a Banach space under the norm

$$
\|f\|_{p}=\lim _{r \rightarrow 1} M_{p}(r, f) .
$$

For $0<p<1$, this is not a norm, but $H^{p}$ is still a complete metric space with a translation invariant metric

$$
d(f, g)=\|f-g\|_{p}^{p}
$$

A linear functional $\varphi$ on $H^{p}$ is bounded $\left(\varphi \in\left(H^{p}\right)^{*}\right)$ if

$$
\|\varphi\|=\sup _{\|f\|_{p}=1}|\varphi(f)|<\infty .
$$

It is easily verify that $\left(H^{p}\right)^{*}$ is a Banach space. Duren, Romberg and Shield (see [4]) were the first to study the linear space structure of the $H^{p}$ space with $0<p<1$. These $H^{p}$ spaces are not Banach spaces and are not locally convex.

They may be regarded as closed subspaces of $L_{p}$ for $0<p<1$; however,it is interesting to note that although there are no continuous linear functionals on $L_{p}$ for $0<p<1$, there are many on $H^{p}$. Duren, Romberg and Shield (see [4]) prove for $1 / 2<p<1$, that $\left(H^{p}\right)^{*}=\Lambda_{\alpha}$ the Lipschitz space of order $\alpha=\frac{1}{p}-1$. For $p \leq \frac{1}{2}$, the results are similar. Even though $H^{p}$ is not locally convex, there are still enough linear functionals to distinguish elements. For example as noted in [4], $g(z)=(1-\xi z)^{-1}$ generates the functional $\varphi(f)=f(\xi)$.

Later, the study of $H^{p}$ spaces was extended to $H^{p}\left(\mathbf{R}^{n}\right)$. The results were highly specialized to $\mathbf{R}^{n}$ until Latter (see [5]), Coifman and Weiss (see [3]) defined $H^{p}\left(\mathbf{R}^{n}\right)$ using the notion of an atom and proved that the 
atomic $H^{p}\left(\mathbf{R}^{n}\right)$ space were equivalent to the original $H^{p}\left(\mathbf{R}^{n}\right)$. Roughly speaking, an atom is a "building block" function which is supported on a ball, has zero integral and has a bounded average.

By thinking of the $H^{p}$ spaces in terms of atoms Coifman and Weiss (see [2]) were able to prove that the dual of $H^{p}$ is again a Lipschitz space of order $\alpha=\frac{1}{p}-1$ not only in $\mathbf{R}^{n}$, but on any homogeneous space $\mathcal{X}$. The $H^{p}$ space for $0<p \leq 1$ on $\mathbf{R}^{n}$ were first characterized in terms of atoms by Coifman (see [3]) and Latter (see [6]). Coifman and Weiss (see [2]) then used this characterization to define $H^{p}(\mathcal{X})$, where $\mathcal{X}$ is a homogeneous space.

In this paper, we extend the work of Coifman and Weiss (see [3]) by defining new Hardy-type spaces using atoms on homogeneous space which we call $H^{\varphi, q}$. The main result of this paper is the following.

Theorem 1.1. Suppose $\varphi$ and $w$ are related by

$$
w^{-1}(t)=\frac{t}{\varphi\left(\frac{1}{t}\right)}
$$

or equivalent by

$$
\varphi(t)=\frac{1}{t w^{-1}\left(\frac{1}{t}\right)}
$$

Suppose also that $\frac{\varphi(t)}{t}$ is a decreasing function of $t$ and that $\frac{\varphi(t)}{t^{\epsilon}}$ is an increasing function for some $0<\epsilon<1$. Let $1 \leq q<\infty$, and let $p$ be conjugate of $q$. Then the dual of $H^{\varphi, q}$ is $B M O_{\varphi}^{p}$.

\section{Atoms}

We begin by defining atoms. The idea for the relationship between $w$ and $\varphi$ functions come from Janson's paper (see [5]). Throughout this paper, we will assume that the measure $\mu$ is a regular measure.

Definition 2.1. A measurable function $a$ is said to be a $(\varphi, q)$ atom if it satisfies:

1. The support of $a$ is contained in a ball $B\left(x_{0}, r\right)$,

2. $\int a d \mu=0$,

3. $\left(\frac{1}{\mu(B)} \int_{B}|a|^{q} d \mu\right)^{\frac{1}{q}} \leq w^{-1}\left(\frac{1}{\mu(B)}\right)$, 
where $w$ and $\varphi$ are related by

$$
w^{-1}(t)=\frac{1}{\varphi\left(\frac{1}{t}\right)} \quad \text { or } \quad \varphi(t)=\frac{1}{t w^{-1}\left(\frac{1}{t}\right)} .
$$

Note that

$$
w^{-1}\left(\frac{1}{\mu(B)}\right)=\frac{1}{\mu(B) \varphi(\mu(B))}
$$

and that (3) can be written as

$$
\|a\|_{q} \leq \mu(B)\left(w^{-1}\left(\frac{1}{\mu(B)}\right)\right)^{q},
$$

where $B=B\left(x_{0}, r\right)$.

\section{Spaces of Homogeneous type}

Let us begin by recalling the notion of space of homogeneous type.

Definition 3.1. A quasimetric $d$ on a set $\mathcal{X}$ is a function $d: \mathcal{X} \times \mathcal{X} \rightarrow$ $[0, \infty)$ with the following properties:

1. $d(x, y)=0$ if and only if $x=y$.

2. $d(x, y)=d(y, x)$ for all $x, y \in \mathcal{X}$.

3. There exists a constant $K$ such that

$$
d(x, y) \leq K[d(x, z)+d(z, y)],
$$

for all $x, y, z \in \mathcal{X}$.

A quasimetric defines a topology in which the balls

$$
B(x, r)=\{y \in \mathcal{X}: d(x, y)<r\}
$$

form a base. These balls may be not open in general; anyway, given a quasimetric $d$, is easy to construct an equivalent quasimetric $d^{\prime}$ such that the $d^{\prime}$-quasimetric balls are open (the existence of $d^{\prime}$ has been proved by using topological arguments in [7]). So we can assume that the quasimetric balls are open. A general method of constructing families $\{B(x, \delta)\}$ is in terms of a quasimetric. 
Example 3.1. A space of homogeneous type $(\mathcal{X}, d, \mu)$ is a set $\mathcal{X}$ with a quasimetric $d$ and a Borel measure $\mu$ finite on bounded sets such that, for some absolute positive constant $A$ the following doubling property holds

$$
\mu(B(x, 2 r)) \leq A \mu(B(x, r))
$$

for all $x \in \mathcal{X}$ and $r>0$.

Next, we are ready to give some example of a space of homogeneous type.

Example 3.2. Let $\mathcal{X} \subset \mathbf{R}^{n}, \mathcal{X}=\{0\} \cup\{x:|x|=1\}$, put in $\mathcal{X}$ the euclidean distance and the following measure $\mu$ : $\mu$ is the usual surface measure on $\{x:|x|=1\}$ and $\mu(\{0\})=1$. Then $\mu$ is doubling so that $(\mathcal{X}, d, \mu)$ is a homogeneous space.

Example 3.3. In $\mathbf{R}^{n}$, let $C_{k}(k=1,2, \cdots)$ be the point $\left(k^{k}+1 / 2,0, \cdots, 0\right)$, for $k \geq 2$, let $B_{k}$ be the ball $B\left(C_{k}, 1 / 2\right)$ and $B_{1}=B(0,1 / 2)$. Let $\mathcal{X}=$ $\cup_{k=1}^{\infty} B_{k}$ with the euclidean distance and the measure $\mu$ such that $\mu\left(B_{k}\right)=$ $2^{k}$ and on each ball $B_{k}, \mu$ is uniformly distributed.

Claim 1. $\mu$ satisfies the doubling condition. Let $B_{r}=B(P, r)$ with $P=$ $\left(P_{1}, \ldots, P_{n}\right)$ and $r>0$.

Case 1. Assume for some $k, B_{k} \subset B_{r}$ and let $k_{0}=\max \left\{k: B_{k} \subset B_{r}\right\}$. Then certainly $P_{1}+r \leq b_{k_{0}+1}=\left(k_{0}+1\right)^{k_{0}+1}+1$ and $\mu\left(B_{r}\right) \geq 2^{k_{0}}$. But, then

$$
\begin{aligned}
P_{1}+2 r & \leq 2\left(\left(k_{0}+1\right)^{k_{0}+1}+1\right) \\
& \leq\left(k_{0}+2\right)^{k_{0}+2}=a_{k_{0}+2} .
\end{aligned}
$$

Therefore $B_{2 r} \subset B_{a_{k_{0}+2}}(0) \equiv B_{0}$. But

$$
\mu\left(B_{0}\right)=\sum_{k=0}^{k_{0}+1} 2^{k} \leq 2^{k_{0}+2} \leq 4 \mu\left(B_{r}\right) .
$$

Hence the doubling condition holds with $A=4$.

Case 2. If for all $k, B_{k} B_{r}$, then $r<1$ so that $B_{r}$ and $B_{2 r}$ intersect only on ball $B_{k}$. Then the doubling condition holds. 


\section{4. $\varphi$-Lipschitz space}

We define the $\varphi$-Lipschitz space and denoted it by $\mathcal{L}_{\varphi}$ to be the space for all measurable functions $f$ on $\mathcal{X}$ for which

$$
|f(x)-f(y)| \leq C \varphi(\mu(B)),
$$

where $B$ is any ball containing both $x$ and $y$ and $C$ is a constant depending only of $f$.

Let $\gamma(f)$ be the inf over all $C$ for which the above inequality holds. Then if we define

$$
\|f\|_{\varphi}^{\mathcal{L}}=\left\{\begin{array}{lll}
\gamma(f) & \text { if } & \mu(\mathcal{X})=\infty \\
\gamma(f)+\int_{\mathcal{X}} f d \mu & \text { if } & \mu(\mathcal{X})=1
\end{array}\right.
$$

a straightforward argument shows that $\mathcal{L}_{\varphi}$, with this norm, is a Banach space. To simplify calculations, we assume that if $\mu(\mathcal{X})$ is finite, then $\mu(\mathcal{X})=1$. We now define $H^{\varphi, q}$ to be the subspace of $\left(\mathcal{L}_{\varphi}\right)^{*}$ consisting of those linear functionals admitting an atomic decomposition as follows:

$h \in H^{\varphi, q}$ if $h$ can be written as a sum $h=\sum_{j \in N} \lambda_{j} a_{j}$, where $a_{j}$ is a $(\varphi, q)$ atom, and $\sum_{j \in N} w\left(\left|\lambda_{j}\right|\right)<\infty$. We denote by the symbol $\|h\|_{\varphi, q}$ the quantity (which is not, in general a norm)

$$
\|h\|_{\varphi, q}=\inf _{\text {alldescompof } f} w^{-1}\left(\sum_{j \in N} w\left(\left|\lambda_{j}\right|\right)\right) .
$$

Example. If $\varphi(t)=t^{\frac{1}{p}-1}$, then $w(t)=t^{p}$ and $H^{\varphi, q}(\mathcal{X})=H^{p}(\mathcal{X})$.

\section{Functions of Bounded $(\varphi, p)$ mean Oscillation}

In this section, we recall the definition of the space of functions of bounded $(\varphi, p)$ mean oscillation, $B M O_{\varphi}^{(p)}(\mathcal{X})$, where $\mathcal{X}$ is a space of homogeneous type. Let $\varphi$ be a nonnegative function on $[0, \infty)$. A locally $\mu$-integrable function $f: \mathcal{X} \rightarrow \mathbf{R}$ is said to belong to the class $\operatorname{BMO}_{\varphi}^{(p)}(\mathcal{X}), 1 \leq p<\infty$, if

$$
\sup \left(\frac{1}{\mu(B)[\varphi(\mu(B))]^{p}} \int_{B}\left|f(x)-f_{B}\right|^{p} d \mu(x)\right)^{\frac{1}{p}}<\infty .
$$

Where the sup is taken over all balls $B \subset \mathcal{X}$ and

$$
f_{B}=\frac{1}{\mu(B)} \int_{B} f(y) d \mu \text {. }
$$

For more detail on functions of bounded $(\varphi, p)$ mean oscillation see Castillo, Ramos Fernández and Trousselot [1]. 


\section{Quasi-Concavity}

In this section, we study the notion of quasi-concavity, which is the condition that we will need to prove our main result.

Definition 6.1. A non-negative function $\phi$ is said to be quasi-convex if there exists a convex function $A$ and a constant $C>1$ such that

$$
A(t) \leq \phi(t) \leq C A(t) .
$$

Definition 6.2. A function $\psi$ is said to be quasi-concave if there exists a constant $C>1$ and a concave function $M$ such that

$$
C M(C t) \leq \psi(t) \leq M(t)
$$

We will use the following Lemmas to prove that the function $W$ as introduced in the definition of a $(\varphi, q)$ atom is quasi-concave under appropriate conditions on $\varphi$.

Lemma 6.1. Suppose that $\frac{\varphi(x)}{x}$ is a decreasing function of $x$, and suppose also that $\frac{\varphi(x)}{x^{\epsilon}}$ is an increasing function for some $0<\epsilon<1$. Let

$$
\psi(x)=\int_{0}^{x} \frac{\varphi(t)}{t} d t .
$$

Then $\psi$ is concave, $\varphi$ is quasi-concave, and $x \psi(x)$ is quasi-convex.

Proof: The derivative

$$
\psi^{\prime}(x)=\frac{\varphi(x)}{x}
$$

is decreasing by hypothesis. Therefore, $\psi$ is concave. To show that $\varphi$ is quasi-concave, first note that $\varphi(x) \leq \psi(x)$ since

$$
\psi(x)=\int_{0}^{x} \frac{\varphi(t)}{t} d t \geq \int_{0}^{x} \frac{\varphi(x)}{x} d t=\varphi(x) .
$$

To show the other inequality, we estimate $\psi(C x)$, for $C<1$ by

$$
\begin{aligned}
\psi(C x) & =\int_{0}^{C x} \frac{\varphi(t)}{t} d t=\int_{0}^{C x} \frac{\varphi(t)}{t^{\epsilon} t^{1-\epsilon}} d t \leq \frac{\varphi(x)}{x^{\epsilon}} \int_{0}^{C x} t^{\epsilon-1} d t \\
& =\frac{\varphi(x)}{x^{\epsilon}} \frac{(C x)^{\epsilon}}{\epsilon}=\varphi(x) \frac{C^{\epsilon}}{\epsilon} .
\end{aligned}
$$


Therefore, we have

$$
\frac{\epsilon}{C^{\epsilon}} \psi(x) \leq \varphi(x)
$$

Next, we choose $C$ by letting $C=\epsilon^{\frac{1}{\epsilon+1}}$.

Since $0<\epsilon<1, C$ also satisfies $C<1$ and $C \psi(C x) \leq \varphi(x)$. Thus, we have shown that $\varphi$ is quasi-concave.

To show that $x \psi(x)$ is quasi-convex, let $g(x)=x \psi(x)$. Note that $\frac{g(x)}{x}=$ $\psi(x)$ is increases, so

$$
A(x)=\int_{0}^{x} \frac{g(u)}{u} d u
$$

is convex. Also,

$$
A(x)=\int_{0}^{x} \frac{g(u)}{u} d u \leq \frac{g(x)}{x} x=g(x),
$$

so $A(x) \leq g(x)$.

We also have

$$
A(2 x)=\int_{0}^{2 x} \frac{g(u)}{u} d u \geq \int_{x}^{2 x} \frac{g(u)}{u} d u \geq \frac{g(x)}{x} x=g(x),
$$

thus

$$
g(x) \leq A(2 x) \leq 2 A(2 x),
$$

and we have shown that

$$
A(x) \leq g(x) \leq 2 A(2 x)
$$

Therefore, $g$ is quasi-convex, which completes the proof.

Lemma 6.2. $\quad$ 1. $\varphi$ is quasi-concave if and only if there exists a constant $C<1$ such that

$$
\frac{\varphi\left(t_{1}\right)}{t_{1}} \geq \frac{C \varphi\left(C t_{2}\right)}{t_{2}}
$$

for all $0 \leq t_{1} \leq t_{2}$

2. $\psi$ is quasi-convex if and only if there exists a $C>1$ such that

$$
\frac{\varphi\left(t_{1}\right)}{t_{1}} \geq \frac{C \varphi\left(C t_{2}\right)}{t_{2}}
$$

for all $0<t_{1} \leq t_{2}$. 
Proof: of $(1)(\Rightarrow)$ since $\varphi$ is quasi-concave, we have $M$ concave and $C<1$ such that

$$
C M(C t) \leq \varphi(t) \leq M(t)
$$

Now, $\frac{M(t)}{t}$ is a non-increasing function of $t$, so, for all $0<t_{1} \leq t_{2}$, we have

$$
\frac{M\left(t_{1}\right)}{t_{1}} \geq \frac{M\left(t_{2}\right)}{t_{2}}
$$

Thus,

$$
\begin{aligned}
\frac{\varphi\left(t_{1}\right)}{t_{1}} & \geq \frac{C M\left(C t_{1}\right)}{t_{1}} \geq \frac{C^{2} M\left(C t_{1}\right)}{C t_{1}} \geq \frac{C^{2} M\left(C t_{2}\right)}{C t_{2}} \\
& =\frac{C M\left(C t_{2}\right)}{t_{2}} \geq \frac{C \varphi\left(C t_{2}\right)}{t_{2}} .
\end{aligned}
$$

$(\Leftarrow)$ Let $C<1, t_{1} \leq t_{2}$, and suppose $\frac{\varphi\left(t_{1}\right)}{t_{1}} \geq \frac{C \varphi\left(C t_{2}\right)}{t_{2}}$. Consider the function

$$
\psi(t)=\frac{1}{C} \int_{0}^{\frac{t}{C}} \inf _{x<s<\frac{t}{C}} \frac{\varphi(s)}{s} d x .
$$

Then $\psi$ is concave by Lemma 6.1 . Also, as in the proof of Lemma 1 , we have

$$
\begin{gathered}
C \psi(t)=\int_{0}^{\frac{t}{C}} \frac{\varphi(x)}{x} d x=\int_{0}^{\frac{t}{C}} \frac{\varphi(x)}{x^{\epsilon} x^{1-\epsilon}} d x \\
\leq \frac{\varphi\left(\frac{t}{C}\right)}{\left(\frac{t}{C}\right)^{\epsilon}}\left(\frac{t}{C}\right)^{\epsilon}=\varphi\left(\frac{t}{C}\right) .
\end{gathered}
$$

Thus, $C \psi(C t) \leq \varphi(t)$, which gives us the first inequality in the quasiconcavity definition. For the other inequality, note that since $\frac{\varphi(t)}{t}$ decreases, and $C<1$,

$$
\psi(t)=\int_{0}^{\frac{t}{C}} \frac{\varphi(x)}{x} d x \geq \int_{0}^{t} \frac{\varphi(x)}{x} d x \geq \varphi(t) .
$$

Thus, we have shown that $C \psi(C t) \leq \varphi(t) \leq \psi(t)$, where $\psi$ is concave, proving that $\varphi$ is quasi-concave.

The proof of (2) is similar to the above proof of (1). 


\section{Duality}

Theorem 1.1 and its proof are modeled on $H^{\varphi, q}$, where $\varphi(t)=t^{1 / p-1}$ and $w(t)=t^{p}$. Clearly, in this case, $\frac{\varphi(t)}{t}$ decreases and $\frac{\varphi(t)}{t^{\epsilon}}$ increases for some $0<\epsilon<1$. To prove Theorem 1.1, we let $L$ be a bounded linear functional on $H^{\varphi, q}$, and we fix a ball $B$ in $\mathcal{X}$. We show first that $L$ is a bounded linear functional on the subspace

$$
L_{0}^{q}(B)=\left\{f \in L^{q}(B): \int_{B} f d \mu=0\right\}
$$

of $L^{q}(B)$. Then, using the Hahn-Banach Theorem and the Riesz Representation Theorem, we extend $L$ to $L^{q}(B)$ with the same norm, and we uniquely represent $L$ by an integral with $L^{p}$ function $g$. Using an increasing sequence of balls converging to $\mathcal{X}$, we then find a unique function $g$ such that if $f \in L^{q}(B)$,

$$
L f=\int_{B} f g d \mu,
$$

for any ball $B$. Finally, by making a $(\varphi, q)$ atom from $f-f_{B}$, we show that $g \in B M O_{\varphi}^{(p)}$, and we note that by Hölder Inequality, $B M O_{\varphi}^{(p)} \subset B M O_{\varphi}$.

To show that any $g \in B M O_{\varphi}^{(p)}$ defines a bounded linear functional on $H^{\varphi, q}$, we first show for an atom $a \in H^{\varphi, q}$, supported on a ball $B$,

$$
\left|\int_{B} \operatorname{gad} \mu\right| \leq\|g\|_{B M_{\varphi}^{(p)}}
$$

for $h \in H^{\varphi, q}$, we decompose $h$ into a sum of $(\varphi, q)$-atoms and we use the quasi-concavity of $w$ to show that

$$
\left|\int_{B} g h d \mu\right| \leq\|g\|_{B M O_{\varphi}^{(p)}} w^{-1}\left(\frac{2}{C^{4}}\|h\|_{H^{\varphi, q}}\right) .
$$

Therefore, $g$ defines a bounded linear functional on $H^{\varphi, q}$ given by

$$
L_{g}(H)=\int g h d \mu
$$

and

$$
\|L\| \leq C\|g\|_{B M O_{\varphi}^{(p)}} .
$$

This shows that $L_{g}$ is a bounded linear functional on $H^{\varphi, q}$. 
Proof of Theorem 1.1 The proof of this Theorem follows along the same lines as the proof of [2]. Let $L$ be a bounded linear functional on $H^{\varphi, q}$, and let $\|L\|$ be the norm of $L$. Fix a ball $B$ in $\mathcal{X}$. Let

$$
L_{0}^{q}(B)=\left\{f \in L^{q}(B): \int_{B} f d \mu=0\right\} .
$$

It follows that if $f \in L_{0}^{q}(B)$, then

$$
a(x)=\frac{[\mu(B)]^{1 / q}}{\|f\|_{L^{q}(B)}} w^{-1}\left(\frac{1}{\mu(B)}\right) f(x)
$$

is a $(\varphi, q)$ atom, since, by $(2)$ of the atomic definition

$$
\begin{aligned}
\left(\frac{1}{\mu(B)} \int_{B}|a(x)|^{q} d \mu(x)\right)^{\frac{1}{q}} & \leq \frac{[\mu(B)]^{1 / q}}{[\mu(B)]^{1 / q}\|f\|_{L^{q}(B)}} w^{-1}\left(\frac{1}{\mu(B)}\right)\|f\|_{L^{q}(B)} \\
& \leq w^{-1}\left(\frac{1}{\mu(B)}\right) .
\end{aligned}
$$

We also have

$$
\|f\|_{H^{\varphi}, q} \leq \frac{1}{w^{-1}\left(\frac{1}{\mu(B)}\right)} \frac{\|f\|_{L^{q}(B)}}{[\mu(B)]^{1 / q}} .
$$

Hence, $L f$ is defined and

$$
\|L f\| \leq\|L\| \frac{\|f\|_{L^{q}(B)}}{[\mu(B)]^{1 / q}} .
$$

That is, $L$ is a bounded linear functional on $L_{0}^{q}(B)$. By the Hahn-Banach Theorem, we can extend $L$ to $L^{q}(B)$ with the same norm and by the Riesz Representation Theorem, we can conclude that there exists $g \in L^{p}(B)$ such that $L f=\int_{B} f g d \mu$ for all $f \in L_{0}^{q}(B)$.

The function $g$ is uniquely determined up to a constant, or, equivalently if $\int_{B} f g d \mu=0$ for all $f \in L_{0}^{q}(B)$, then it follows that $g$ is a constant. To see this, suppose $\int_{B} f g d \mu=0$ for all $f \in L_{0}^{q}(B)$. Choose $h \in L^{q}(B)$.

Since $h-h_{B} \in L_{0}^{q}(B)$, we have

$$
0=\int_{B} g\left(h-h_{B}\right) d \mu=\int_{B}\left(g h-g h_{B}\right) d \mu=\int h\left(g-g_{B}\right) d \mu .
$$

Since this equality holds for all $h \in L^{q}(B)$, it must be true that $g(x)=g_{B}$ a.e. $x$ in $B$. 
Let $\left\{B_{j}\right\}_{j=1}^{\infty}$ be an increasing sequence of balls converging to $\mathcal{X}$, such that $\mu\left(B_{1}\right)>0$. We obtain a function $\tilde{g}_{j}$ satisfying

$$
L f=\int_{B_{j}} f \tilde{g}_{j} d \mu
$$

for each $j$. Now, let

$$
g_{j}=\tilde{g}_{j}-\left(\tilde{g}_{j}\right)_{B_{1}} .
$$

Then, $\int_{B_{1}} g_{j} d \mu=0$. It remains to show that $\left.g_{j}\right|_{B_{k}}=g_{k}$ for all $k \leq j$. By the above remark, we know that on $B_{k} \supset B_{1}$, we have $g_{j}-g_{k}=C$. Now, integrate both sides over $B_{1}$ to obtain

$$
\int_{B_{1}}\left(g_{j}-g_{k}\right) d \mu=\int_{B_{1}} C d \mu
$$

which implies that $0=\mu\left(B_{1}\right)$. Therefore, $C=0$, and we conclude that $\left.g_{j}\right|_{B_{k}}=g_{k}$ for $k \leq j$.

We now have a unique function $g$ such that if $f \in L^{q}(B)$, then

$$
L f=\int_{B} f g d \mu,
$$

which holds for any ball $B$.

In particular, if $a$ is a $(\varphi, q)$ atom supported in $B$, we have

$$
\|L\| \geq|L a|=\left|\int_{B} g a d \mu\right|=\left|\int_{B}\left(g-g_{B}\right) a d \mu\right|,
$$

if $f$ is supported in $B$ and $\|f\|_{L^{q}}=1$, then

$$
a=\frac{[\mu(B)]^{1 / q}}{2} w^{-1}\left(\frac{1}{\mu(B)}\right)\left(f-f_{B}\right)
$$

is a $(\varphi, q)$ atom, since

$$
\begin{aligned}
\left(\frac{1}{\mu(B)} \int_{B}|a(x)|^{q} d \mu\right)^{\frac{1}{q}} & =\frac{[\mu(B)]^{1 / q}}{2[\mu(B)]^{1 / q}} w^{-1}\left(\frac{1}{\mu(B)}\right)\left(\int_{B}\left|f-f_{B}\right|^{q} d \mu\right)^{\frac{1}{q}} \\
& \leq \frac{w^{-1}\left(\frac{1}{\mu(B)}\right)}{2} 2\left(\int_{B}|f|^{q} d \mu\right)^{\frac{1}{q}} \\
& \leq w^{-1}\left(\frac{1}{\mu(B)}\right)
\end{aligned}
$$


Now, using this atom in (7.2) above, and using the fact that $g-g_{B}$ has mean zero on $B$, we obtain

$$
\left|\int_{B}\left(g-g_{B}\right) \frac{[\mu(B)]^{1 / q}}{2} w^{-1}\left(\frac{1}{\mu(B)}\right)\left(f-f_{B}\right) d \mu\right| \leq\|L\|,
$$

which implies that

$$
\left|\int_{B}\left(g-g_{B}\right) \frac{[\mu(B)]^{1 / q}}{2} w^{-1}\left(\frac{1}{\mu(B)}\right) f d \mu\right| \leq\|L\|,
$$

which in turn implies that

$$
\left|\int_{B}\left(g-g_{B}\right) f d \mu\right| \leq \frac{2\|L\|}{[\mu(B)]^{1 / q} w^{-1}\left(\frac{1}{\mu(B)}\right)} .
$$

If we now take the supremum of all $f$ supported in $B$ such that $\|f\|_{L^{q}}=$ 1 , we obtain

$$
\begin{aligned}
\left\|g-g_{B}\right\|_{L^{p}} & \leq \frac{2\|L\|}{[\mu(B)]^{1 / q} w^{-1}\left(\frac{1}{\mu(B)}\right)} \\
& =\frac{2\|L\|}{[\mu(B)]^{1 / q}} \mu(B) \varphi(\mu(B)) \\
& =2\|L\|[\mu(B)]^{1 / q} \varphi(\mu(B)) .
\end{aligned}
$$

Rewriting this inequality, we obtain

$$
\left(\frac{1}{\mu(B)[\varphi(\mu(B))]^{p}} \int_{B}\left|g-g_{B}\right|^{p} d \mu\right)^{\frac{1}{p}} \leq 2\|L\|,
$$

so $g \in B M O_{\varphi}^{(p)}$. By Hölder's inequality, we also have

$$
\begin{aligned}
\frac{1}{\varphi(\mu(B)) \mu(B)} \int_{B}\left|g(x)-g_{B}\right| d \mu(x) & \leq \frac{[\mu(B)]^{1 / q}}{\mu(B) \varphi(\mu(B))}\left(\int_{B}\left|g-g_{B}\right|^{p} d \mu\right)^{\frac{1}{p}} \\
& \leq\left(\frac{1}{\mu(B)[\varphi(\mu(B))]^{p}} \int_{B}\left|g-g_{B}\right|^{p} d \mu\right)^{\frac{1}{p}} .
\end{aligned}
$$

So $g \in B M O_{\varphi}$, also.

We have now shown that

$$
\left(H^{\varphi, q}\right)^{*} \subset B M O_{\varphi}^{(p)} \subset B M O_{\varphi} .
$$


Now, suppose that $g \in B M O_{\varphi}^{(p)}$. We will show that $g$ defines a bounded linear functional on $H^{\varphi, q}$. Let $a$ be a $(\varphi, q)$ atom. Then

$$
\begin{aligned}
\left|\int_{B} g a d \mu\right| & =\left|\int_{B}\left(g-g_{B}\right) a(u) d \mu\right| \leq \int\left|g-g_{B}\right| a(u) d \mu \\
& \leq\left(\int_{B}\left|g-g_{B}\right|^{p} d \mu\right)^{\frac{1}{p}}\left(\int_{B}|a(u)|^{q} d \mu\right)^{\frac{1}{q}} \\
& \leq\left(\int_{B}\left|g-g_{B}\right|^{p} d \mu\right)^{\frac{1}{p}}[\mu(B)]^{1 / q} w^{-1}\left(\frac{1}{\mu(B)}\right) \\
& \leq \frac{[\mu(B)]^{1 / q}}{\mu(B) \varphi(\mu(B))}\left(\int_{B}\left|g-g_{B}\right|^{p} d \mu\right)^{\frac{1}{p}} \\
& \leq\left(\frac{1}{\mu(B)[\varphi(\mu(B))]^{p}} \int_{B}\left|g-g_{B}\right|^{p} d \mu\right)^{\frac{1}{p}} \\
& \leq\|g\|_{B M O_{\varphi}^{(p)}}
\end{aligned}
$$

Therefore, we have shown that

$$
\left|\int_{B} \operatorname{gad} \mu\right| \leq\|g\|_{B M O_{\varphi}^{(p)}}
$$

Now, let $h \in H^{\varphi, q}$ and let $h=\sum_{j=1}^{\infty} \alpha_{j} a_{j}$ be decomposition of $h$ into $(\varphi, q)$ atom such that

$$
w^{-1}\left(\sum w\left(\left|\alpha_{j}\right|\right)\right) \leq\left(1+C^{4}\right)\|h\|_{H^{\varphi, q}},
$$

where $C<1$ is the quasi-concavity constant for $w$. Since $C<1$ then

$$
\begin{aligned}
\sum\left|\alpha_{j}\right| & \leq w^{-1}\left(\frac{1}{C} \sum w\left(\frac{\left|\alpha_{j}\right|}{C}\right)\right) \\
& \leq w^{-1}\left(\frac{1}{C} \sum w\left(\frac{C\left|\alpha_{j}\right|}{C^{2}}\right)\right) .
\end{aligned}
$$

Now, let $t_{1}=\left|\alpha_{j}\right|$, and let $t_{2}=\frac{\left|\alpha_{j}\right|}{C^{2}}$. Since $C<1$, we have $t_{1}<t_{2}$ and by Lemma 6.2 implies that

$$
C \frac{w\left(C t_{2}\right)}{t_{2}} \leq \frac{w\left(t_{1}\right)}{t_{1}}
$$

Therefore, we have

$$
w\left(C t_{2}\right) \leq \frac{t_{2}}{t_{1}} \frac{w\left(t_{1}\right)}{C}
$$


which implies that

$$
w\left(C \frac{\left|\alpha_{j}\right|}{C^{2}}\right) \leq \frac{\left|\alpha_{j}\right|}{C^{2}\left|\alpha_{j}\right|} \frac{w\left(\left|\alpha_{j}\right|\right)}{C}=\frac{w\left(\left|\alpha_{j}\right|\right)}{C^{3}} .
$$

Thus

$$
\begin{aligned}
\sum\left|\alpha_{j}\right| & \leq w^{-1}\left(\frac{1}{C} \sum \frac{w\left(\left|\alpha_{j}\right|\right)}{C^{3}}\right)=w^{-1}\left(\frac{1}{C^{4}} \sum w\left(\left|\alpha_{j}\right|\right)\right) \\
& \leq w^{-1}\left(\frac{1}{C^{4}}\left(1+C^{4}\right)\|h\|_{H^{\varphi, q}}\right) \\
& \leq w^{-1}\left(\frac{2}{C^{4}}\|h\|_{H^{\varphi, q}}\right) .
\end{aligned}
$$

Now, if $g \in B M O_{\varphi}^{(p)}$, since $C<1$, we have

$$
\begin{aligned}
& \left|\int g h d \mu\right| \leq \sum\left|\alpha_{j}\right|\left|\int g a_{j} d \mu\right| \\
& \leq\|g\|_{B M O_{\varphi}^{(p)}} w^{-1}\left(\frac{2}{C^{4}}\|h\|_{H^{\varphi}, q}\right) .
\end{aligned}
$$

Therefore, $g$ defines a bounded linear functional $L$ on $H^{\varphi, q}$ given by

$$
L_{g}(h)=\int g h d \mu
$$

which satisfies

$$
\left\|L_{g}\right\|=\sup _{\|h\|_{H^{\varphi}, q=1}}\left|L_{g}(h)\right| \leq w^{-1}\left(\frac{2}{C^{4}}\right)\|g\|_{B M O_{\varphi}^{(p)}} .
$$

Thus,

$$
B M O_{\varphi}^{(p)} \subset\left(H^{\varphi, q}\right)^{*}
$$

and the Theorem is proved. 


\section{References}

[1] Castillo, R. E., Ramos Fernández, J. C. and Trousselot, E. Functions of Bounded $(\varphi, p)$ Mean Oscillation. Proyecciones Journal of Math. Vol 27, No. 2, pp. 163-177, August (2008).

[2] Coifman, R. R, A real variable characterization of $H^{p}$. Studia Math. 51, pp. 269-274, (1974).

[3] Coifman, R. R and Weiss, G. Extensions of Hardy spaces and their use in Analysis. Bull. Amer. Math. Soc. 83, pp. 99-157, (1977).

[4] Duren, P. L., Romberg, B. W. and Shields, A. L., Linear functionals on $H^{p}$ spaces with $0<p<1$. J. Reine Angew, Math 238, pp. 32-60, (1969).

[5] Janson, S. Generalization of Lipschitz spaces and an application to Hardy space and bounded mean oscillation. Duke Math. 47, pp. 959982, (1980).

[6] Latter, R. A. A characterization of $H^{p}\left(\mathbf{R}^{n}\right)$ in terms of atoms. Shidia Math 62 (1978) pp. 93-101, (1978).

[7] Macias, R. and Segovia, C. Lipschitz functions on space of homogeneous type, Avd. Math., 33, pp. 257-270, (1979).

\section{René Erlín Castillo}

Departamento de Matemáticas,

Universidad Nacional de Colombia

AP360354 Bogotá

e-mail : recastillo@unal.edu.co

\section{Julio C. Ramos Fernández}

Departamento de Matemáticas, Universidad de Oriente 6101 Cumaná, Edo. Sucre, Venezuela

e-mail : jcramos@udo.edu.ve 
and

Eduard Trousselot

Departamento de Matemáticas,

Universidad de Oriente 6101 Cumaná,

Edo. Sucre,

Venezuela

e-mail : eddycharles2007@hotmail.com 